(the log-rank $\mathrm{p}=0.047$ ), while those who had normal ECG had a significantly lower mortality (the log-rank $p=0.003$ ). In conclusion, centenarians who have major ECG abnormality had higher levels of NT-proBNP and higher mortality, while those who exhibited normal ECG had survival advantage.

\section{BEHAVIORAL THERAPIES FOR INSOMNIA IMPROVE NOCTURIA IN OLDER WOMEN VETERANS}

C. Fung 2,1, C.A. Alessi²,1, Y. Song ${ }^{1}$, C. Pedigo-Vaughn ${ }^{3,4}$, A. Markland ${ }^{5,6}$, A. Huang7, S. McGowan ${ }^{2}$, J. Martin', 1. University of California, Los Angeles, Los Angeles, California, 2. VA Greater Los Angeles, Los Angeles, California, 3. Atlanta VA, Decatur, Georgia, 4. Emory University, Atlanta, Georgia, 5. Birmingham VA Medical Center, Birmingham, Alabama, 6. University of Alabama, Birmingham, Alabama, 7. University of California, San Francisco, San Francisco, California

Background: Nocturia is common in older women with chronic insomnia disorder. Few studies have examined nocturia symptoms and treatments in older women veterans. This study assessed changes in nocturia frequency associated with behavioral interventions for insomnia in older women veterans. Methods: In the context of an ongoing comparative effectiveness trial of two behavioral interventions for insomnia conducted at a VA medical center among women veterans, data from baseline, post-intervention, and 3-month follow up for participants aged $\geq 55$ years were analyzed. Measures included self-reported nocturia frequency in the past month $(0-5+/$ night $)$ and degree of bother from nocturia (4-point scale; $0=$ not at all, $3=\mathrm{a}$ lot). Nocturia frequency responses were categorized as nocturia ( $\geq 2$ times/night) versus no nocturia $(<2$ times/night). Differences in the proportion of participants with nocturia and degree of bother (post-intervention vs. baseline; 3-month follow-up vs. baseline) were examined with twosample test of proportions and t-tests, respectively. Results: 36 participants (mean age 63, range 55-91 years) completed all or part of their assigned intervention. At baseline, 23 (64\%) participants had nocturia. Nocturia decreased post-intervention (11 [33\%], p=.011) and at 3-month follow-up (7 [25\%], $\mathrm{p}=.002$ vs. baseline). Degree of bother mean scores decreased from baseline to post-intervention (1.43 vs. $0.75, \mathrm{p}=.014$ ) and 3 -month follow-up (1.56 vs. 0.89, p=.031). Conclusions: Nocturia frequency and degree of bother improve with behavioral interventions for insomnia. These results suggest that a sleep-focused, non-pharmacological strategy for treating nocturia may be effective and indicate a need for a trial examining such a strategy.

\section{MORTALITY AND ASSOCIATED RISK FACTORS IN COMMUNITY-DWELLING PERSONS WITH EARLY DEMENTIA}

B. Barrett ${ }^{1,2}$, T. Bulat ${ }^{1,2}$, S.K. Schultz ${ }^{1}$, S. Luther ${ }^{1}$, 1. HSR \&D Center of Innovation on Disability and Rehabilitation Research, Department of Veterans Affairs, Tampa, Florida, 2. Patient Safety Center of Inquiry, Department of Veterans Affairs, Tampa, Florida

This study examined mortality in relation to baseline characteristics amongst participants in a longitudinal study of persons diagnosed with early dementia $(\mathrm{N}=143)$. Department of Veterans Affairs' health records were used to collect participant mortality outcomes, for a standardized six-year observation period. Study measures assessed participant: demographics; functioning (Inventory of Activities of Daily Living; Tinetti Gait and Balance); cognitive- (MiniMental State Examination [MMSE], Trails-Making Test B) and dementia-related (Clinical Dementia Rating [CDR]) factors; as well as medication use (categorized into cholinesterase-inhibitors and confusion-related medications]). Bivariate analyses examined associations with baseline characteristics and mortality. A series of Cox proportional hazard models were conducted examining associations with baseline variables, both in univariate as well as multivariable models (controlling for age and gender, where appropriate). Over the six years of study follow-up, $48.3 \%$ of participants died. The mean time to death was 2.98 years $(S D=1.44)$, with a minimum of 54 days and a maximum of 5.91 years. Across all univariate models, only the model including the MMSE was statistically significant. Multivariable model results indicated female gender and higher cognition (as measured by the MMSE) were associated with a reduced likelihood of death, controlling for other factors. This study examined mortality in persons with early dementia living in the community, and associations with baseline factors. Cognitive decline was significantly associated with mortality, even amongst persons early in the course of dementia. Findings underscore the terminal nature of dementia, frequently underappreciated by family and caregivers in the community.

\section{HEALTHSPAN MEASURES IN A WESTERN DIET CONDITIONED, IRRADIATED RODENT MODEL OF ACCELERATED AGING}

K. Fanning, C. Sherrill, A. Davis, K. Kavanagh, Pathology,

Wake Forest School of Medicine, Winston-Salem, North Carolina

Deteriorating metabolic and physical function play significant roles in declining healthspan, however most animal models of aging rely on single gene mutations and few demonstrate translationally relevant multi-system decline. This study is the first to compare healthspan measures (metabolic and physical function) in rodents on a high-fat diet exposed to sub-lethal doses of whole body irradiation (IR). Four week old male and female C57/BL6 mice consuming a western diet (17\% protein, $35 \%$ fat, $48 \%$ carbohydrate) were randomized as IR or control (CTL). At 8 weeks, IR mice received 7 Gy total body irradiation (Cobalt60, divided as 2 fractions delivered at $357 \mathrm{rad} / \mathrm{min}$ ), with metabolic assessments evaluated at early (4 weeks) and late (12-16 weeks) post-exposure time-points ( $\mathrm{n}=6 / \mathrm{group} /$ time-point). Delayed effects of irradiation included significantly higher fasting glucose at both time-points $(\mathrm{p} \leq 0.01)$ and worse insulin sensitivity (AUCITT; $\mathrm{p}=0.10$ ) with comparable body weights between groups. Physical function outcomes were assessed late, with IR resulting in functional deficits that were $23 \%$ lower on average across 6 function tests ( $n=8-12 /$ group). IR mice scored significantly worse on the 4-limb hang time test measuring muscular endurance $(\mathrm{p}=0.02)$ and on a composite performance score $(\mathrm{p}<0.05)$. Measures were co-varied by body weight which was comparable between groups. As hypothesized, IR mice demonstrated poorer glucose metabolism and physical function relative to control non-irradiated mice. This work adds support to the use of IR mice as a model of accelerated aging. 